\title{
On the relation between action selection and movement control in 5- to 9-month-old infants
}

\author{
Margot van Wermeskerken · John van der Kamp • \\ Geert J. P. Savelsbergh
}

Received: 16 November 2010/ Accepted: 17 March 2011/Published online: 2 April 2011

(C) The Author(s) 2011. This article is published with open access at Springerlink.com

\begin{abstract}
Although 5-month-old infants select action modes that are adaptive to the size of the object (i.e., oneor two-handed reaching), it has largely remained unclear whether infants of this age control the ensuing movement to the size of the object (i.e., scaling of the aperture between hands). We examined 5-, 7-, and 9-month-olds' reaching behaviors to gain more insight into the developmental changes occurring in the visual guidance of action mode selection and movement control, and the relationship between these processes. Infants were presented with a small set of objects (i.e., 2, 3, 7, and $8 \mathrm{~cm}$ ) and a large set of objects (i.e., 6, 9, 12, and $15 \mathrm{~cm}$ ). For the first set of objects, it was found that the infants more often performed two-handed reaches for the larger objects based on visual information alone (i.e., before making contact with the object), thus showing adaptive action mode selection relative to object size. Kinematical analyses of the twohanded reaches for the second set of objects revealed that inter-trial variance in aperture between the hands decreased with the approach toward the object, indicating that infants' reaching is constrained by the object. Subsequent
\end{abstract}

M. van Wermeskerken $(\bowtie)$. J. van der Kamp

G. J. P. Savelsbergh

Research Institute MOVE, Faculty of Human Movement

Sciences, VU University, Van der Boechorststraat 9,

1081 BT Amsterdam, The Netherlands

e-mail: mm.van.wermeskerken@gmail.com

J. van der Kamp

Institute of Human Performance, University of Hong Kong,

Hong Kong SAR, China

G. J. P. Savelsbergh

Institute for Biomedical Research into Human Movement

and Health, Manchester Metropolitan University,

Manchester, UK analysis showed that between hand aperture scaled to object size, indicating that visual control of the movement is adjusted to object size in infants as young as 5 months. Individual analyses indicated that the two processes were not dependent and followed distinct developmental trajectories. That is, adaptive selection of an action mode was not a prerequisite for appropriate aperture scaling, and vice versa. These findings are consistent with the idea of two separate and independent visual systems (Milner and Goodale in Neuropsychologia 46:774-785, 2008) during early infancy.

Keywords Infant reaching - Action mode selection . Movement control · Bimanual reaching · Aperture scaling · Two-visual systems model

\section{Introduction}

When reaching out for an object, adults select action modes that are adaptive to properties of the object such as size, shape, and orientation. For example, large objects are reached for and grasped with two hands instead of one hand, and pronated or supinated grasps are used to match the object's orientation (Rosenbaum et al. 1992; Stelmach et al. 1994; van der Kamp et al. 1998). These action modes set the boundaries within which the ensuing movement toward the object must be controlled. For example, the aperture between thumb and index finger (i.e., in the case of a one-handed reach) or between the right and left index fingers (i.e., in the case of a two-handed reach) is scaled to the size of the object, the maximal apertures being larger for larger objects (Jeannerod 1981; Marteniuk et al. 1990; Smeets and Brenner 1999; Stelmach et al. 1994; Tresilian and Stelmach 1997). Indeed, movement control is qualitatively the same for one-handed and two-handed reaches, 
although quantitative differences between these action modes exist (Tresilian and Stelmach 1997). That is, although the maximal aperture of a two-handed reach is scaled to object size, it is larger and more variable compared to the maximal aperture of a one-handed reach. This implies that despite movement control being adaptive to object size in both one-handed and two-handed reaching, it is specific for different action modes. Indeed, it turns out that action mode selection and movement control are separate processes that have distinct properties, at least as far as the role of vision is concerned (e.g., Glover 2004; Milner and Goodale 2008; Woodworth 1899). Van Doorn and colleagues (van Doorn et al. 2007), for instance, demonstrated that allocentric information sources (i.e., context-dependent, relative metrics) are exploited for the selection of the action mode, while online movement control relies on egocentric sources of information (i.e., context-independent, absolute metrics). This was attested for by an influence of the Müller-Lyer illusion on the selection of an action mode that was adapted to object length (i.e., the choice to reach one- or two-handed), whereas subsequent movement execution (i.e., hand aperture) appeared immune to the illusion (see also Crajé et al. 2008). The contention of separate visual processes for action mode selection and online movement control is consistent with long standing tradition (e.g., Woodworth 1899), the most recent formulation of which is the twovisual systems model (Glover 2004; Milner and Goodale 2008; see also Dijkerman et al. 2009). Even though the selection of an appropriate action mode and the online control of movement engage in separate visual processesat least in adults - it is pertinent that they seamlessly interact (van der Kamp et al. 2008). For instance, at certain aperture widths participants choose to rotate their shoulders when walking through the aperture at fast speeds, but not when walking at a normal speed (Warren and Whang 1987).

The present study portrays the adaptive action mode selection (i.e., the selection of one- and two-handed reaches) and movement control (i.e., aperture scaling) in relation to object size in 5- to 9-month-old infants. We specifically aim to chart age-related changes in these two processes to uncover how they might mutually constrain each other during early development.

Studies of early infant reaching and grasping suggest large variations in onset ages of adaptive action mode selection and movement control relative to object size. Some authors reported that infants' action mode selection is not adapted to object size before 8 months of age (Corbetta et al. 2000; Corbetta and Snapp-Childs 2009; Fagard 2000; Fagard and Pezé 1997; see also Corbetta and Thelen 1996). However, these studies have used relatively large objects that might have discouraged one-handed reaches. In contrast, studies that presented smaller objects have reported compelling evidence that infants as young as 3-5 months select an appropriate action mode related to object size (Bruner and Koslowski 1972; Newell et al. 1989a, 1993; Siddiqui 1995; van Hof et al. 2002). Indeed, Newell and colleagues showed that infants from 4 months of age select two-handed grasps when the ratio between the size of the hand and the size of the object exceeds a critical value, while for lower values a one-handed grasp is used (Newell et al. 1993; see also Newell et al. 1989a, b). Yet, there is some controversy as to whether the early emergence of adaptive action mode selection among the 4-month-olds is uniquely based upon visual information or also involves tactile information from object contact (see Corbetta et al. 2000; Corbetta and Snapp-Childs 2009).

With respect to movement control, infants use visual information to online guide their reaching and grasping movements at least from 4 to 5 months of age (Bushnell 1985; von Hofsten and Fazel-Zandy 1984; von Hofsten and Roennqvist 1988). For instance, infants adjust their hand orientation to the orientation of the object prior to contact. Yet, research thus far has failed to show that hand aperture is scaled to object size before 9 months of age (Fagard 2000; von Hofsten and Roennqvist 1988). Thus, von Hofsten and Roennqvist (1988) reported that 5-month-old infants did not show an increase in aperture with increasing object size, neither did Fagard (2000) for 5- and 7-montholds. However, in both studies, hand aperture was determined by measuring the distance or angle between thumb and index finger of one hand. Yet, hand aperture measurement is taxing in 5- and 7-month-olds, because they have tiny fingers and tend to make fairly erratic arm movements with many rotations. Moreover, von Hofsten and Roennqvist suggested that 5-month-olds might not have detected the small differences in the size of the objects (i.e., objects with diameters of 1.5, 2.5, and $3.5 \mathrm{~cm}$ ). Although Fagard (2000) used larger objects, she measured aperture between thumb and index finger of one hand, which is not necessarily adjusted to object size in case of two-handed attempts during which the object is grasped between fingers of both hands. Consequently, the conclusion that movement control is not tuned to object size before 9 months of age may reflect an artifact of the experimental procedures rather than a lack of ability in younger infants.

Yet, some have recently concluded that taken together these findings point to a developmental delay in movement control processes relative to action mode selection processes (e.g., Barrett et al. 2008; Fagard 2000). This conclusion may be somewhat premature, but does raise the question whether the two processes follow distinct developmental trajectories, and relatedly, to what degree the 
development of either process constrains the development of the other.

Hence, the current study aims at directly assessing this developmental relation, by investigating 5- to 9-month-old infants' visually guided action mode selection and movement control while reaching for different-sized objects. In doing so, we will first examine whether infants are capable of selecting an action mode (i.e., one- versus two-handed reaching) that is appropriate for object sizes varying between $2 \mathrm{~cm}$ and $8 \mathrm{~cm}$ based on visual information alone. Subsequently, we assessed visual control of movement by presenting the infants a second series of larger objects (i.e., between $6 \mathrm{~cm}$ and $15 \mathrm{~cm}$ ), for which the ratio between the size of the hand and the size of the object presumably exceeds the critical value for one-handed reaches, in order to enhance the occurrence of two-handed reaches. The particular twist is that these larger objects allow us to examine scaling of the aperture between the two hands, which potentially eliminates many difficulties related to measuring aperture between thumb and index finger and allows larger variations in object size, which presumably are easier to detect by the infants. Finally, we aim to establish the developmental relationship between action mode selection and movement control processes, by exploring whether infants who show adaptive action mode selection also demonstrate better aperture scaling and vice versa.

\section{Methods}

\section{Participants}

Thirteen 5-month-old $(M=5.08$ months, $\mathrm{SD}=.10), 16$ 7-month-old $(M=6.97$ months, $\quad \mathrm{SD}=.10), \quad$ and 10 9-month-old ( $M=9.05$ months, $\mathrm{SD}=.17$ ) healthy fullterm infants participated in the study after their parents gave written informed consent. Fourteen additional infants (5 5-month-olds, 3 7-month-olds, and 6 9-month-olds) were tested but excluded from the analysis because of fussing, crying, not reaching or experimental failure (i.e., too much missing kinematical data). The local institution's ethical committee approved the experiment.

Apparatus and task

Infants were seated in a specially designed infant seat with adjustable supports such that infants had their trunk straight, head upright, and limbs free to move. The seat was reclined at $18^{\circ}$ from the vertical. The experiment consisted of two parts in which two different series of objects were presented to the infants. During the first part, infants were presented with four polystyrene, multicolored balls with diameters of $2,3,7$, and $8 \mathrm{~cm}$, which were presented to the infant on a height-adjustable tripod by the use of a magnet. The tripod was positioned such that the attached objects were within reach of the infant. During the second part of the experiment, infants were presented with a second series of four polystyrene, multicolored balls with diameters of 6 , 9,12 , and $15 \mathrm{~cm}$. To enhance flexibility in presentation (particularly for the two larger objects, which more easily dropped when presented on the tripod) these objects were presented on the experimenter's right hand with the palm facing up and the object resting on top of it. The object was slightly secured by the thumb at the back and the middle finger at the front of the object in order to leave the infant enough space to grasp the object.

Infants' reaching and grasping movements were recorded with a high-speed camera (Basler A602f, Basler AG, Arhensburg, Germany) that sampled at $100 \mathrm{~Hz}$. During the second part of the experiment, also a 3-D motion analysis system (Optotrak 3020, Northern Digital Inc., Waterloo, Ontario, Canada) recorded infants' reaching movements with a sampling frequency of $200 \mathrm{~Hz}$. Two pre-calibrated Optotrak camera units were positioned at $2.5 \mathrm{~m}$ from the infant on opposing sides of the setup and were calibrated to one another (measurement error $<.1 \mathrm{~mm}$ ). The infants wore bracelets with three infrared light-emitting diodes (IREDs) on each wrist to assure that the entire movement could be tracked also in case of arm rotation. One IRED was attached to the experimenter's right thumb to specify the location of the object. An external trigger synchronized the high-speed recordings and Optotrak measurements. To capture the entire reaching movements of the infant, the objects were presented after the recording was triggered.

\section{Design and procedure}

Once the infant was seated in the chair, the experimenter adjusted the height and distance of the tripod so that an attractive toy (different from the objects used in the study) attached to the tripod was within reach for the infant. The infant was allowed to grasp the toy, while the experimenter fastened the bracelets with the IREDs to the infant's wrists. Then, the experiment started with the first set of objects. In most cases, the four objects were each presented twice in random order. If the infant was distracted, infant's attention was drawn to the object. If the infant did not reach for an object or in case that it was not completely clear as to whether a reach had occurred, this trial was repeated after a block of four object presentations. In case that both the original and the additional trials contained suitable data, all trials were included in the analyses (i.e., for some infants this procedure resulted into 3 instead of 2 presentations per object). Hereafter, the tripod was removed and the experimenter seated in front of the infant, presenting the second 
set of objects on her right hand. Optotrak was switched on to record the infants' arm movements. The same procedure and design were applied for this second set of objects with the exception that the objects were presented three to four times in random order. Each trial was terminated when the infant succeeded in grasping the object or refused to reach for it for approximately $30 \mathrm{~s}$. Together this resulted in 20 to 24 trials (i.e., approximately 8 trials in the first part and about 12 to 16 trials in the second part of the experiment), which took approximately $20 \mathrm{~min}$.

Data analysis

\section{First series of objects}

Behavioral measures Infants' reaching behaviors were scored from the high-speed recordings. It was scored whether infants performed a reach and whether this reach was with one or two hands. A reach was defined as an object-directed arm movement during which the infant is looking at the object and is making contact with the object. A two-handed reach was defined as both hands moving in the direction of the object before either hand contacted it with the infants' gaze directed at the object. If one hand approached the object or if one hand contacted the object before the other started to move, the reach was coded as a one-handed reach.

For each infant and each object, the proportion of onehanded reaches was submitted to a 3 (age: 5, 7, 9 months) $\times 4$ (object size: $2,3,7,8 \mathrm{~cm}$ ) analysis of variance with repeated measures on the last factor (RMANOVA). Since the proportion variables were not distributed normally, these variables underwent an arcsine transformation before statistical testing.

For this and subsequent analyses of variance, we report Hyun-Feldt adjustments of the $P$ values in cases that the sphericity assumption was violated (i.e., epsilon's $>1.0$ ). All post hoc tests were conducted using the Bonferroni adjustment $(P<.05)$.

\section{Second series of objects}

Behavioral measures For this second part of the experiment, the same behavioral measures were obtained as in the first part of the experiment. Again, the proportion of one-handed reaches was submitted to a 3 (age: 5, 7, 9 months) $\times 4$ (object size: $6,9,12,15 \mathrm{~cm}$ ) analysis of variance with repeated measures on the last factor. To normalize the data, the proportion variables underwent an arcsine transformation before statistical testing.

Kinematic measures The 3-D positions of the IREDs given by the Optotrak motion analysis system were filtered with a second-order recursive Butterworth low-pass filter with a cutoff frequency of $10 \mathrm{~Hz}$. For the reaching movements, data of one of the three IREDs with most data across trials were selected for further analysis. Still, some trials showed intervals with missing data, which were interpolated using a least squares method over gaps no longer than $100 \mathrm{~ms}$. Trials in which the gap with missing data exceeded this $100 \mathrm{~ms}$ were excluded from further kinematic analysis. Subsequently, movement onset (i.e., the moment at which either hand started to move in the direction of the object) and the end of the movement (i.e., the moment at which both hands were contacting the object or lifted the object from the experimenter's hand) were determined by using the multiple sources of information method (see Schot et al. 2010). This method has proven to be very robust in movement segmentation in adult reaching and entails that several objective functions are formulated that together compute the likelihood that a certain instant in time is the start or end of the movement. The moment with the highest likelihood is then taken as the moment of movement onset (i.e., independently determined for each hand) or end of the movement (for a detailed description of the objective functions for the determination of movement onset and the end of the movement see "Appendix"). Since infants' reaching movements are much more variable than adults', the starting and end points resulting from this method were compared to the video recordings. In a few cases, these moment were clearly too late or too early and were adjusted accordingly.

Subsequently, a reach was classified as two-handed in the case that both hands were moving to the object before either hand contacted it and in case that the difference in movement onset between both hands was less than $500 \mathrm{~ms}$. Otherwise, the reach was classified as a one-handed reach and excluded from further analysis. We then determined, for each instant in time, the distance between the two hands (i.e., wrist IREDs); this will be referred to as between hand aperture.

We first tried to establish that infants indeed controlled the between hand aperture during the reach and then show that this control is related to object size. That is, in case that between hand aperture is controlled during the reach, trialto-trial variation in between hand aperture should diminish during the approach toward the object, reflecting homing in on the object (Bootsma and van Wieringen 1990). Thus, for each infant and each object size, the inter-trial variance in between hand aperture was determined between $0 \mathrm{~cm}$ and $15 \mathrm{~cm}$ from the endpoint with intervals of $.5 \mathrm{~cm}$. Subsequently, the variance in between hand aperture was submitted to a repeated measures ANOVA with object size and distance as within-subjects factor and age as betweensubjects factor.

Thereafter, we examined whether between hand aperture was scaled to object size. It is pertinent that in adults it has been found that aperture at reach onset affects maximal 
hand aperture (Hesse and Deubel 2009; Timmann et al. 1996; Wallace et al. 1990). Infants, however, show large variability in how they execute their (two-handed) reach. For instance, at reach onset, hands may be opened and arms fully extended sideward and maximally separated (Newman et al. 2001; von Hofsten and Roennqvist 1988). Hence, maximal between hand aperture might not be the most reliable variable, and we therefore also computed for each infant and each object size the mean between hand aperture at the distances of 0 to $15 \mathrm{~cm}$ before the endpoint with intervals of $.5 \mathrm{~cm}$.

Because in most reaches the arms did not start moving synchronously, we determined aperture between the wrists and its variance at the moment that the leading hand was at a certain distance from the endpoint (i.e., irrespective of the position of the other hand).

The mean maximal between hand aperture was submitted to a 3 (age: 5, 7, 9 months) $\times 4$ (object size: $6,9,12,15 \mathrm{~cm}$ ) analysis of variance with repeated measures on the last factor. In addition, the mean between hand aperture per object size was submitted to a repeated measures ANOVA with distance and object size as within-subjects factor and age as between-subject factor. All dependent variables were distributed normally and had equal variances.

Relation between action mode selection and movement control Finally, we examined the relation between action mode selection and movement control. That is, for each individual infant and each reach it was determined, for the first set of objects, whether they selected an adaptive action mode. An action mode was coded as adaptive in the case that the two smallest objects (i.e., 2 and $3 \mathrm{~cm}$ ) were approached unimanually and the two larger objects of the first set of objects (i.e., 7 and $8 \mathrm{~cm}$ ) were approached bimanually. However, if the infant successfully lifted the 7- or 8-cm object from the tripod with one hand, this was also coded as adaptive. ${ }^{1}$ Subsequently, the proportion of adaptive action modes was computed, and based on a median-split, each infant was classified as adaptive selector or less-adaptive selector.

For the second set of objects, the Pearson product correlation between hand aperture at $2.5 \mathrm{~cm}$ before the endpoint and object size were computed for each individual infant. $^{2}$ In case of a significant correlation $(P<.05)$, the

\footnotetext{
${ }^{1}$ This only occurred for the older infants who have slightly bigger hands than the younger infants and sometimes were able to grasp the 7-cm object with one hand.

${ }^{2}$ We chose to correlate between hand aperture at $2.5 \mathrm{~cm}$ before the endpoint to object size, rather than at a different (larger) distance, because, as will be shown below, the trial-to-trial variance in between hand aperture is least at this distance and, hence, between hand aperture is more controlled at this distance than at larger distances before the endpoint. Moreover, at this distance the infant had not contacted the object yet.
}

infant was categorized as a scaler; otherwise, the infant was classified as a non-scaler.

We then examined whether there were age-related effects in the relative occurrence of adaptive versus less-adaptive selectors and scalers versus non-scalers using a chi-square test. Finally, it was investigated whether there were more adaptive selectors among the scalers compared to the nonscalers (and vice versa) by using a chi-square test.

\section{Results}

First series of objects

In the first part of the experiment, infants were presented with objects with diameters of 2, 3, 7, and $8 \mathrm{~cm}$. Each object was presented approximately twice, resulting in a total of 323 reaches, of which $88 \%$ were successful (i.e., lifting the object from the tripod; Table 1). A RM-ANOVA on the proportion of one-handed reaches for each age group and object size revealed that with increasing object size, infants increasingly performed two-handed reaches $(F(3,108)=130.8 ; \quad P<.001 ;$ see Fig. 1a). Post hoc comparisons revealed that the two smallest objects elicited significantly more one-handed reaches than the two largest objects $(P<.001)$. No interaction effect with age was observed, which indicates that selecting one or two hands was similarly affected by object size across age groups.

\section{Second series of objects}

In the second part of the experiment, infants were presented with the second set of objects (i.e., 6, 9, 12, and $15 \mathrm{~cm})$. Each object was presented three or four times, which resulted in a total of 573 reaches, of which $83 \%$ were successful (Table 1). An RM-ANOVA revealed a significant effect of object size on the proportion of onehanded reaches, which indicates that more two-handed reaches were performed with increasing object size $(F(3,108)=7.5 ; P<.01$; see Fig. $1 b)$. Post hoc analyses showed that the $6-\mathrm{cm}$ object was more frequently reached for with one hand compared to the other objects (all $P$ 's $<.05)$. However, it should be noted that even the 6-cm object was reached for bimanually in $83 \%$ of the cases. There was neither a significant age effect, nor a significant interaction effect between object size and age. Hence, the effect of object size on two-handed reaching was similar for the different age groups.

\section{Kinematic analysis}

We first examined whether between hand aperture was constrained by the object, because this can be taken as an 
Table 1 Number of object presentations and percentage of successful reaches per object size and age group

\begin{tabular}{|c|c|c|c|c|c|c|}
\hline \multirow[t]{2}{*}{ Object size $(\mathrm{cm})$} & \multicolumn{2}{|c|}{ 5-month-old $(n=13)$} & \multicolumn{2}{|c|}{ 7-month-old $(n=16)$} & \multicolumn{2}{|c|}{ 9-month-old $(n=10)$} \\
\hline & Presentations & Success $(\%)$ & Presentations & Success $(\%)$ & Presentations & Success $(\%)$ \\
\hline 2 & 28 & 67.9 & 33 & 87.9 & 20 & 95.0 \\
\hline 3 & 27 & 74.1 & 32 & 96.9 & 20 & 100.0 \\
\hline 7 & 27 & 81.5 & 35 & 85.7 & 20 & 95.0 \\
\hline 8 & 27 & 92.6 & 34 & 88.2 & 20 & 95.0 \\
\hline 6 & 41 & 87.8 & 59 & 96.6 & 37 & 100 \\
\hline 9 & 44 & 86.4 & 64 & 93.8 & 38 & 92.1 \\
\hline 12 & 45 & 66.7 & 65 & 80.0 & 39 & 82.1 \\
\hline 15 & 40 & 47.5 & 63 & 81.0 & 38 & 71.1 \\
\hline
\end{tabular}
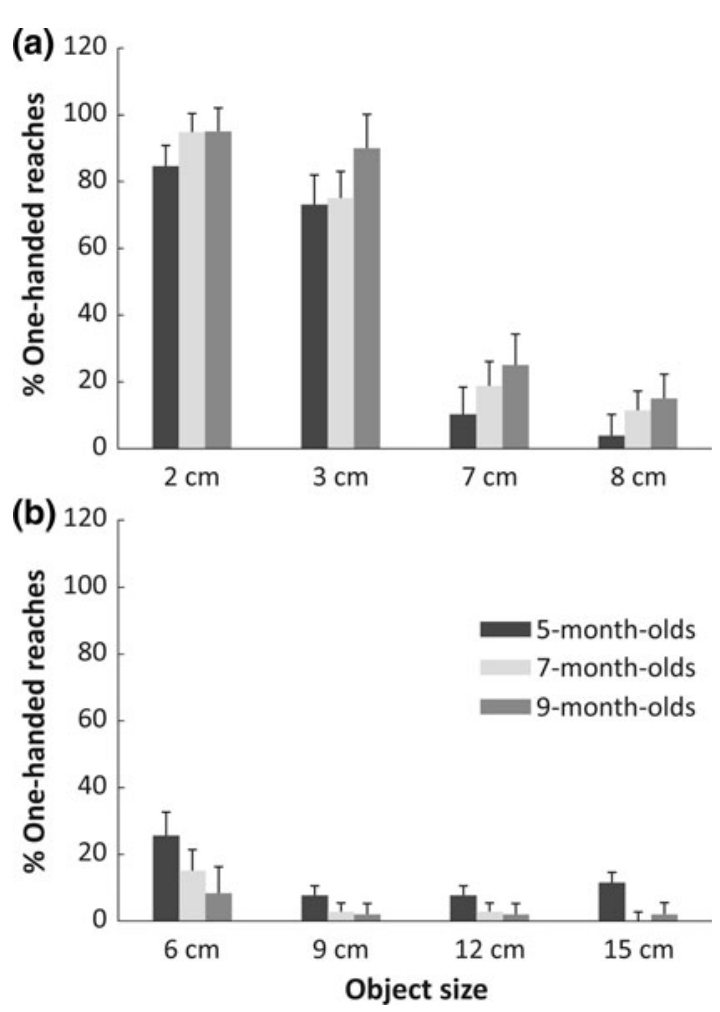

Fig. 1 The percentage of one-handed reaches for each object size and age group for the first set of objects (a) and second set of objects (b). Error bars represent standard error

indicator of control. To this end, we calculated the average individual inter-trial variation in between hand aperture from the two-handed reaches toward the second series of objects. Infants who did not perform a two-handed reach toward all object sizes $(n=7)$ were not taken into account. Moreover, analyses were performed on infants who contributed at least two reaches in which both hands covered a distance of at least $7 \mathrm{~cm}$ in each object condition. More specifically, 7 5-month-olds, 13 7-month-olds, and 8 9-month-olds were included. On average, each infant performed 13-14 reaches that were classified as two-handed and in which both hands covered a distance of $7 \mathrm{~cm}$, resulting in a total of 387 reaches. The average distance covered during a reach was $19 \mathrm{~cm} \pm 5.6 \mathrm{~cm}$.

In Fig. 2, the trial-to-trial variance in between hand aperture is depicted for distances up to $15 \mathrm{~cm}$ from the endpoint. It can be seen that initially up to $7 \mathrm{~cm}$ from the endpoint the inter-trial variation remains relatively constant. Yet, at shorter distance, from $7 \mathrm{~cm}$ up to the endpoint, the variation gradually, but consistently, decreases. To verify that indeed inter-trial variation in between hand aperture decreased, we compared the trial-to-trial variance in between hand aperture for 10 distance intervals before the endpoint (i.e., $2.5-7 \mathrm{~cm}$ in steps of $.5 \mathrm{~cm}$ ). ${ }^{3}$ Distance intervals up to $2.5 \mathrm{~cm}$ were chosen, because within this distance infants were likely to contact the object, yielding additional tactile information to control reaching and grasping. Moreover, we did not consider longer distances than $7 \mathrm{~cm}$ from the endpoint to maximize the number of reaches and infants included in the analyses. As a result, the variance in between hand aperture was submitted to a 3 (age: 5, 7, 9 months) $\times 4$ (object size: 6, 9, 12, $15 \mathrm{~cm}) \times 10$ (distance: $2.5,3,3.5,4,4.5,5,5.5,6,6.5$, $7 \mathrm{~cm}$ ) analysis of variance with repeated measures on the latter two factors. This revealed a significant effect of distance $(F(9,225)=10.9, P<.001)$. Post hoc analyses indicated that the inter-trial variation of between hand aperture decreased when getting closer to the object (direct comparison revealed significant effects between 2.5 and distances up to $6 \mathrm{~cm}$, between 3 and $5,5.5$, and $6 \mathrm{~cm}$, and between 3.5 and 5.5 and $6 \mathrm{~cm}$ (all $P$ 's $<.05)$ ). Neither a main effect of object size nor a main effect of age was observed $(P>.1)$. All interaction effects were non-

\footnotetext{
${ }^{3}$ In some cases (i.e., $15 \%$ of the trials) it occurred that one of the hands or both started within a distance of $7 \mathrm{~cm}$ of the object. This trial was only considered for the distances traveled. For instance, if one of the hands or both moved $5.6 \mathrm{~cm}$, hand aperture was determined for $2.5,3,3.5,4,4.5,5$ and $5.5 \mathrm{~cm}$ before the endpoint. For the larger distances (i.e., $6,6.5$, and $7 \mathrm{~cm}$ ) this trial was excluded.
} 
significant. These findings show that infants' reaching is increasingly constrained during the approach toward the object. This homing in suggests that control of aperture between the two hands is already apparent from at least 5 months of age.

Figure 3 shows between hand aperture as function of object size and age. It is apparent from this figure that maximal between hand aperture occurs early during the reach and is not affected by object size. This was confirmed by a RM-ANOVA that was conducted on the mean maximal between hand aperture as function of age group and object size. This did not reveal any significant effects (all $P$ 's $>.1$ ). Hence, in 5- to 9-month-olds maximal between hand aperture was not controlled relative to object size. This was not surprising given the previously reported large differences in aperture between the hands at reach onset (see also Newman et al. 2001; von Hofsten and Roennqvist 1988). However, as can be seen in Fig. 3, it appears that for all age groups between hand aperture is scaled to object size from approximately $7 \mathrm{~cm}$ up to the endpoint. Hence, to further clarify whether between hand aperture was constrained by object size prior to contact, we compared between hand aperture for each age group and object size at ten distance intervals before the endpoint (i.e., $2.5 \mathrm{~cm}$ to $7 \mathrm{~cm}$ in steps of .5). This 3 (age: 5, 7, 9 months) $\times 4$ (object size: $6,9,12,15 \mathrm{~cm}$ ) $\times 10$ (distance: $2.5,3,3.5,4$, $4.5,5,5.5,6,6.5,7 \mathrm{~cm})$ RM-ANOVA revealed a main effect of distance $(F(9,225)=305.2, P<.001)$ and object size $(F(3,75)=23.1, P<.001)$. Post hoc comparisons revealed that when infants home in on the object (i.e., distance between the hands and object decreases), between hand aperture gets significantly smaller (all $P$ 's $<.001$ ). Moreover, infants' aperture between hands increased with object size; i.e., the largest objects (i.e., 12 and $15 \mathrm{~cm}$ ) yielded a significant larger between hand aperture than the two smallest objects (i.e., 6 and $9 \mathrm{~cm}$ ). There was no main effect of age. However, a significant interaction effect between object size and distance was revealed $(F(27,675)=2.9, \quad P<.05), \quad$ which indicates that

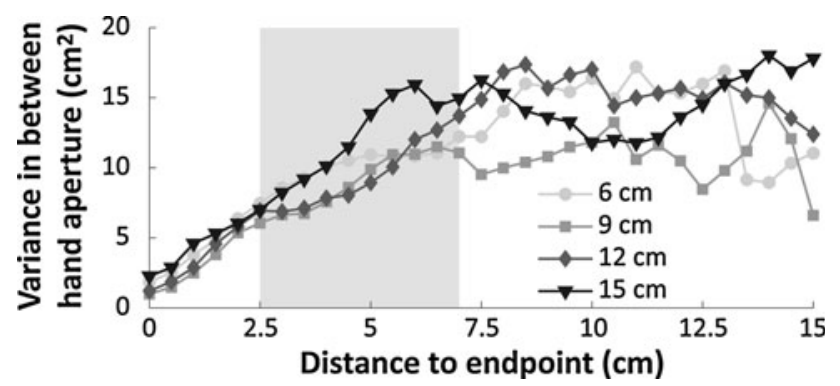

Fig. 2 Mean variance in between hand aperture across infants plotted against the distance before the endpoint. Note that statistical tests were performed for distances in the shaded area depicted in the figure differences in between hand aperture for each object size become more pronounced during the approach toward the object (but before it is contacted). Together these findings indicate that infants as young as 5 months scale their between hand aperture to object size prior to contact.

Relation between action mode selection and movement control

To complete the analysis, we assessed the relation between action mode selection and movement control within individual infants. All infants who performed bimanual reaches for each object size in the second part of the experiment were included in this analysis $(n=32)$.

Infants were categorized as adaptive selectors in case that they selected adaptive action modes in more than $87.5 \%$ of the reaches; otherwise, they were classified as less-adaptive selectors. In addition, infants who yielded a significant correlation between hand aperture and object
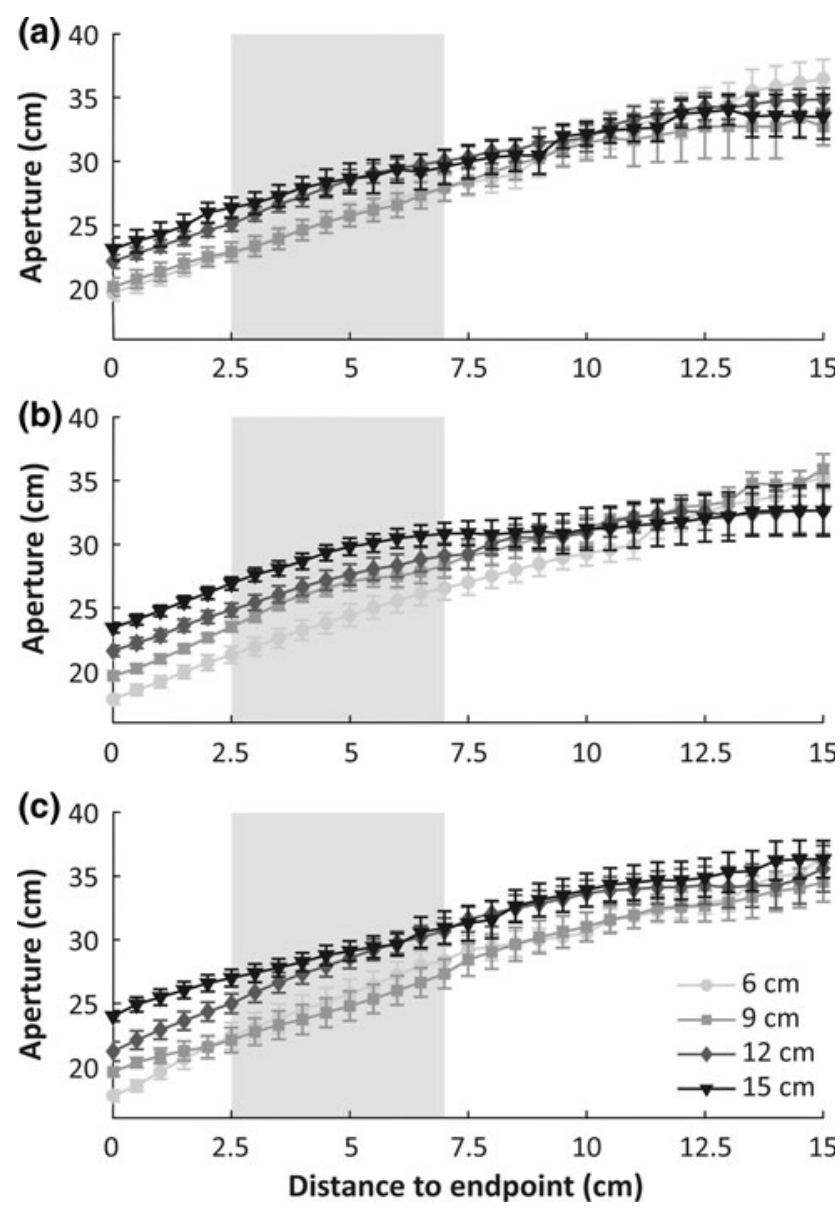

Fig. 3 Between hand aperture at different distances before the endpoint for each object size depicted for a 5-month-olds; b 7-montholds; and c 9-month-olds. Note that statistical tests were performed for distances in the shaded area depicted in the figure. Error bars represent standard error 
size were categorized as scalers (mean correlation $=.69 \pm .13)$; otherwise, infants were coded nonscalers (mean correlation $=.37 \pm .18$ ).

First, a significant chi-square test $\left(\chi^{2}(2, N=32)=6.1\right.$; $P<.05)$ revealed that the number of infants that selected an adaptive action mode increased with age (Table 2). Second, although more infants among the 7- and 9-montholds were categorized as scalers than in 5-month-olds, this effect was not significant $\left(\chi^{2}(2, N=32)=1.1 ; P>.1\right)$. Hence, the relative amount of infants who scaled their between hand aperture to the size of the object did not increase with age (Table 2).

Finally, we examined the relation between less-adaptive selectors and adaptive selectors on the one hand, and nonscalers and scalers on the other hand; the chi-square test did not reveal a systematic relation between the two categories (Table $\left.3 ; \chi^{2}(1, N=32)=.00 ; P=1\right)$. Hence, we found no evidence to support the idea that the two processes are dependent.

\section{Discussion}

Several authors have suggested that there is a developmental delay of movement control relative to action mode selection (e.g., Barrett et al. 2008; Fagard 2000). This contention is based on comparison of observations showing that, on the one hand, infants as young as 3-5 months are able to select adaptive action modes relative to object size during reaching and grasping (Bruner and Koslowski 1972; Newell et al. 1989a, 1993; Siddiqui 1995; van Hof et al. 2002), but on the other hand fail to adjust their ongoing reaching movements to object size before 9 months of age (Fagard 2000; von Hofsten and Roennqvist 1988). However, to date, a direct investigation (i.e., within-individual comparison) into the developmental relation between these two processes was lacking. The present study, therefore, aimed at exploring this relation by mapping the development of 5- to 9-month-olds' action mode selection and movement control during reaching. We restricted our analyses to visual guidance by exclusively focusing on reaching behaviors prior to object contact. This precludes contributions of tactile information, which has been contentious particularly with respect to action mode selection
Table 3 Distribution of infants that were classified as adaptive or less-adaptive selectors and scalers or non-scalers

\begin{tabular}{lll}
\hline & Adaptive selector & Less-adaptive selector \\
\hline Scaler & 15 & 5 \\
Non-scaler & 9 & 3 \\
\hline
\end{tabular}

(Corbetta et al. 2000; Corbetta and Snapp-Childs 2009; Newell et al. 1989a, 1993). The current findings indicate that infants as young as 5 months select an action mode that is appropriately adapted to object size and scale the aperture between the hands to the size of the object during the reach based on visual information. No evidence was forthcoming, however, that the processes were dependent on each other. Thus, being able to select an adaptive action mode was not a prerequisite for being able to scale the hand aperture during the reach or vice versa.

Action mode selection and movement control

The finding that infants as young as 5 months adapt their action mode to object size is in line with previous findings that reported adaptive action mode selection between 3 and 5 months of age (Bruner and Koslowski 1972; Newell et al. 1989a, 1993; Siddiqui 1995; van Hof et al. 2002). Moreover, the finding indicates that action mode selection in these young infants is visually guided. The selection of a one- or two-handed reach was adaptive before the infants contacted the object, that is, before tactile information about object size became available. Nonetheless, the present study adds that not all 5-month-olds can be considered as adaptive selectors; instead it shows that the proportion of adaptive selectors increases until 9 months of age. The cause of these individual differences is not clear, although it seems unlikely that they are predicated on their ability to control arm movements (see below).

The current study also shows that infants control their aperture between the hands during the reach. The trialto-trial variance in between hand aperture decreased during the approach toward the object. However, it appears that this variance does not consistently decrease over time. Rather, initially the variance is relatively high and remains roughly constant up to approximately $7 \mathrm{~cm}$ before
Table 2 Number (and percentage) of infants categorized as adaptive or lessadaptive selectors and scalers or non-scalers

\begin{tabular}{|c|c|c|c|c|}
\hline & \multicolumn{2}{|c|}{ Action mode selection } & \multicolumn{2}{|c|}{ Movement control } \\
\hline & Adaptive selector & Less-adaptive selector & Scaler & Non-scaler \\
\hline 5 months & $5(50.0 \%)$ & $5(50.0 \%)$ & $5(50.0 \%)$ & $5(50.0 \%)$ \\
\hline 7 months & $11(78.6 \%)$ & $3(21.4 \%)$ & $10(71.4 \%)$ & $4(28.6 \%)$ \\
\hline 9 months & $8(100 \%)$ & $0(0 \%)$ & $5(62.5 \%)$ & $3(37.5 \%)$ \\
\hline
\end{tabular}


the endpoint. It is not unlikely that this larger variance partly reflects the inter-trial differences in initial hand position that have been reported previously (Newman et al. 2001; von Hofsten and Roennqvist 1988). Yet, late in the reach, at shorter distance, (i.e., within $7 \mathrm{~cm}$ from the endpoint), the trial-to-trial variance decreases. This suggests that between hand aperture is increasingly constrained. Such funnel-like decreases in inter-trial variation during the approach toward an object have been taken as an indicator for visual control (Bootsma and van Wieringen 1990; but see van Soest and Beek 2010). This was already apparent in infants as young as 5 months. We also found that toward the end of the reach, at shorter distances from the object, control of between hand aperture was scaled to object size and that this effect was similar across age groups. Hence, from 5 months onwards, infants scale their between hand aperture to the size of the object prior to contact (i.e., based on visual information). However, individual analyses revealed that not all infants displayed aperture scaling, but that a small majority of the infants (i.e., approx. 60\%) scaled their between hand aperture to the size of the object. Perhaps surprisingly, the proportion of scalers among the infants did not increase with age. Nevertheless, it is pertinent that our findings indicate that movement control is actually adaptive to object size at 5 months. This contradicts previous observations that infants younger than 9 months do not scale aperture to the size of the object (Fagard 2000; von Hofsten and Roennqvist 1988). We believe that several methodological differences explain these discrepancies in onset of online movement control between the studies. It was the current study's focus on two-handed rather than one-handed reaches in particular, which allowed us to uncover early signs of movement control in relation to object size. Before drawing firm conclusions, however, an alternative explanation for the early age of onset of movement control in the present study must be considered. The discrepancy in age onset between the studies might be related to differential involvement in distal and proximal musculature. Indeed, it has been argued that control of the more distal musculature (i.e., involved in finger movements) develops late relative to the control of proximal musculature (i.e., in arm movements; Gesell 1946; Halverson 1931; Kuypers 1962, 1982). Since the present study assessed aperture between the hands rather than between fingers as was done in previous studies, the discrepancy in age of onset of aperture control between the studies may reflect different developmental trajectories for proximal and distal control. It should be noted that the hypothesis of a developmental progression from proximal to distal control uniquely considers organismic maturational constraints, and may underestimate the role of task and environmental constraints on infants' reaching (Newell 1986). In fact, even 6-month-olds employ precision grasps that require (distal) control of the fingertips, but only when presented with very small objects (Lee et al. 2006). Nevertheless, the proximaldistal argument partly explains the differences between the current and previous findings concerning movement control. Importantly, however, the current study shows that at least from 5 months onwards, infants are able to control their reaching movements based on visual information about the size of the object.

Hence, we conclude that at least from 5 months onwards infants display movement control, i.e., control their (bimanual) reaching movements relative to object properties such as size.

Developmental relation between action mode selection and movement control

Together these findings suggest that 5-month-old infants use visual information to adaptively select an action mode relative to object size and scale their reaching movements to the size of the object. Yet, no systematic developmental relationship was revealed between the two processes. That is, infants who were able to select an adaptive action mode not necessarily tuned their between hand aperture to the size of the object, and vice versa, infants who scaled between hand aperture to object size were not always adaptive selectors. This indicates that between 5 and 9 months of age the processes are not necessarily dependent. Moreover, there were age differences for action mode selection (i.e., with increasing age more infants selected adaptive action modes) but not for movement control. This implies that the two processes may follow separate developmental trajectories. These observations are consistent with infant catching of balls that approach at different speeds. The choice whether or not to perform a catching movement (i.e., action selection) did follow a different developmental trajectory than the changes in the timing of the catching movement (i.e., movement control; van Hof et al. 2008). Van Hof et al. did propose that improvements in action selection were predicated on improvements in movement control. Future studies must find out whether a similar relationship between action selection and movement control holds for object size when 3- to 4-month-olds are examined.

\section{Two-visual systems}

The finding that action mode selection and movement control are not necessarily dependent, but likely separate processes is consistent with recent interpretations of the two-visual systems model (Glover 2004; Milner and Goodale 2008; see also Dijkerman et al. 2009). According to this model, there are two functionally and anatomically 
separate systems; the dorsal vision for movement system and the ventral vision for perception system. The contention is that the dorsal system is heavily involved in visual control of movements (i.e., online scaling of aperture to object size), whereas the ventral system is not only engaged in obtaining knowledge of the environment (i.e., perception), but also in selecting and preparing appropriate actions (Dijkerman et al. 2009; van Doorn et al. 2007). The present findings are consistent with the idea that in the majority of infants at the age of 5 months the two-visual systems are already functioning independently (Atkinson 2000; Bertenthal 1996; van der Kamp and Savelsbergh 2000; van Wermeskerken et al. 2010; van Wermeskerken et al. 2011). Yet, it is important to stress that the present study does not prove this independency. Because adult studies show that online movement control and action mode selection primarily rely on egocentric (contextindependent) and allocentric (context-dependent) information sources, respectively, manipulating these sources of information in isolation (e.g., by using visual illusions) should differentially affect infants' movement control and action mode selection, if they indeed function independently. This may also shed more light on the developmental relationship between the two processes.

Acknowledgments We thank the parents and infants for their enthusiastic cooperation. We thank Niek Pot, Ana Smorenburg, and Sascha Haans for their help in conducting the experiment.

Open Access This article is distributed under the terms of the Creative Commons Attribution Noncommercial License which permits any noncommercial use, distribution, and reproduction in any medium, provided the original author(s) and source are credited.

\section{Appendix}

The multiple sources of information method consists of the formulation of multiple objective functions that together compute the likeliness that the start and end of the movement occurs at a certain instant in time (Schot et al. 2010). For each of the objective functions, each instant in time is assigned a value between 0 and 1; the larger the value the larger is the chance that this instant in time represents the start or end of the movement. The instant in time that has the maximum likelihood of being the moment of movement onset and the endpoint of the movement is then determined by multiplying the values of all the objective functions for each instant in time.

We determined the endpoint of the movement with the following objective functions:

1. The distance between hands of the infant and the object should be smaller than 2.5 times the size of the object; otherwise, the infants' hands are positioned too far from the object to be a possible endpoint. We then added a constant value to the size of the object, because unlike for one-handed grasps where the fingers are directly in contact with the object, the aperture between the hands (i.e., wrist IRED's) is larger than the actual hand opening. This function was applied to both hands independently.

$F_{\text {Pos }}\left(\overline{\text { pos }_{\text {hand }} \text { pos }_{\text {object }}} \leq 2.5(s+c)\right)=1$

and

$F_{\text {Pos }}\left(\overline{\operatorname{pos}_{\text {hand }} \text { pos }_{\text {object }}}>2.5(s+c)\right)=0$,

where poshand represents the position of the infant's hand, pos $_{\text {object }}$ represents the position of the object, $s$ is the size of the object, and $c$ is the constant value which was added to object size.

2. The closer the infants' hands are to the object, the more likely it is that this is the endpoint of the movement. For each single trial, the maximal distance $\left(d_{\max }\right)$ between the infant's hand and the object was determined. The smaller the distance between the hand and the object relative to the maximal distance, the more likely it is that this instant in time represents the endpoint. This function was applied to both hands.

$F_{\text {Dist }}=1-\frac{\overline{\text { pos }_{\text {hand }} \text { pos }_{\text {object }}}}{d_{\max }}$

3. The aperture at contact between the hands $(g)$ should be related to the size of the object. The more the aperture resembles the size of the object, the larger the outcome of the objective function:

$F_{\text {Hand }}(g<(s+c))=\frac{g}{(s+c)}$,

$F_{\text {Hand }}((s+c)<g<2(s+c))=\frac{2(s+c)-g}{(s+c)}$,

and

$F_{\text {Hand }}(g \geq 2(s+c))=0$

4. The hand aperture should decrease during the approach toward the object ( $\dot{g}$ represents the derivative of aperture):

$F_{\text {Close }}(\dot{g} \leq 0)=1$

and

$F_{\text {Close }}(\dot{g}>0)=0$

5. Near to the end of the movement (i.e., at contact), the hands should not move at a high speed. Hence, the lower the velocity of the hands $(v)$ relative to peak velocity $\left(v_{\max }\right)$, the larger the chance that a particular instant represents the end of the movement. This function was computed for both hands separately. 


$$
F_{\mathrm{Vel}}=1-\frac{v}{v_{\max }}
$$

6. The hands should be decelerating toward the end of the movement ( $\dot{v}$ represents the derivative of velocity). (In order not to exclude instances in time at which the hands were not decelerating, a value .5 was assigned to these instances instead of 0 .) This function was computed for both hands separately.

$F_{\text {Acc }}(\dot{v} \leq 0)=1$

and

$$
F_{\text {Acc }}(\dot{v}>0)=0.5
$$

The start of the movement was determined for each hand separately. The following objective functions were used:

1. The start of the movement should at least occur $100 \mathrm{~ms}$ before the end of the movement.

$F_{\mathrm{Mt}}=1$ (for moments $100 \mathrm{~ms}$ prior to end point)

and

$F_{\mathrm{Mt}}=0$ (for moments within $100 \mathrm{~ms}$ of endpoint)

2. The start of the movement should occur before peak velocity $\left(v_{\max }\right)$ of the hand.

$F_{\text {MaxVel }}=1$ (for moments prior to peak velocity)

and

$F_{\mathrm{MaxVel}}=0$ (for moments after peak velocity)

3. Hand velocity should be smaller or equal to $5 \mathrm{~cm} / \mathrm{s}$ for at least five concurrent frames:

$F_{\mathrm{Vel}}(v \leq 5)=1$

and

$F_{\text {Vel }}(v>5)=0$

4. The hand should accelerate:

$F_{\text {Acc }}(\dot{v}>0)=1$

and

$F_{\text {Acc }}(\dot{v} \leq 0)=0$.

\section{References}

Atkinson J (2000) The developing visual brain. Oxford University Press, Oxford

Barrett TM, Traupman E, Needham A (2008) Infants' visual anticipation of object structure in grasp planning. Infant Behav Dev 31:1-9
Bertenthal BI (1996) Origins and early development of perception, action, and representation. Annu Rev Psychol 47:431-459

Bootsma RJ, van Wieringen PC (1990) Timing an attacking forehand drive in table tennis. J Exp Psychol Hum Percept Perform 16:21-29

Bruner JS, Koslowski B (1972) Visually preadapted constituents of manipulatory action. Perception 1:3-14

Bushnell EW (1985) The decline of visually guided reaching during infancy. Infant Behav Dev 8:139-155

Corbetta D, Snapp-Childs W (2009) Seeing and touching: the role of sensory-motor experience on the development of infant reaching. Infant Behav Dev 32:44-58

Corbetta D, Thelen E (1996) The development origins of bimanual coordination: a dynamic perspective. J Exp Psychol Hum Percept Perform 22:502-522

Corbetta D, Thelen E, Johnson K (2000) Motor constraints on the development of perception-action matching in infant reaching. Infant Behav Dev 23:351-371

Crajé C, van der Kamp J, Steenbergen B (2008) The effect of the "rod-and-frame" illusion on grip planning in a sequential object manipulation task. Exp Brain Res 185:53-62

Dijkerman HC, McIntosh RD, Schindler I, Nijboer TCW, Milner AD (2009) Choosing between alternative wrist postures: action planning needs perception. Neuropsychologia 47:1476-1482

Fagard J (2000) Linked proximal and distal changes in the reaching behavior of 5-to 12-month-old human infants grasping objects of different sizes. Infant Behav Dev 23:317-329

Fagard J, Pezé A (1997) Age changes in interlimb coupling and the development of bimanual coordination. $\mathrm{J}$ Motor Behav 29:199-208

Gesell A (1946) The ontogenesis of infant behavior. In: Carmichael L (ed) Manual of child psychology. Wiley, New York, pp 295-331

Glover S (2004) Separate visual representations in the planning and control of action. Behav Brain Sci 27:3-78

Halverson HM (1931) An experimental study of prehension in infants by means of systematic cinema records. Genetic Psychol Monogr 10:107-286

Hesse C, Deubel H (2009) Changes in grasping kinematics due to different start postures of the hand. Hum Mov Sci 28:415-436

Jeannerod M (1981) Intersegmental coordination during reaching at natural visual objects. In: Long J, Baddeley A (eds) Attention and performance, IX. Erlbaum, Hillsdale, pp 153-168

Kuypers HGJM (1962) Corticospinal connections: postnatal development in the rhesus monkey. Science 138:678-680

Kuypers HGJM (1982) A new look at the organization of the motor system. In: Kuypers HGJM, Martin GF (eds) Progress in brain research, vol. 57. Descending pathways to the spinal cord. Elsevier Biomedical, Amsterdam, pp 381-404

Lee MH, Liu YT, Newell KM (2006) Longitudinal expressions of infant's prehension as a function of object properties. Infant Behav Dev 29:481-493

Marteniuk RG, Leavitt JL, MacKenzie CL, Athenes S (1990) Functional relationships between grasp and transport components in a prehension task. Hum Mov Sci 9:149-176

Milner AD, Goodale MA (2008) Two visual systems re-viewed. Neuropsychologia 46:774-785

Newell KM (1986) Constraints on the development of action. In: Wade MG, Whiting HTA (eds) Motor development in children: aspects of coordination and control. Martinus-Nijhoff, Dordrecht, pp 341-360

Newell KM, Scully DM, McDonald PV, Baillargeon R (1989a) Task constraints and infant grip configurations. Dev Psychobiol 22:817-831

Newell KM, Scully DM, Tenenbaum F, Hardiman S (1989b) Body scale and the development of prehension. Dev Psychobiol 22:1-13 
Newell KM, McDonald PV, Baillargeon R (1993) Body scale and infant grip configurations. Dev Psychobiol 26:195-205

Newman C, Atkinson J, Braddick O (2001) The development of reaching and looking preferences in infants to objects of different sizes. Dev Psychol 37:561-572

Rosenbaum DA, Vaughan J, Barnes HJ, Jorgensen MJ (1992) Time course of movement planning: selection of hand grips for object manipulation. J Exp Psychol Learn Mem Cogn 18:1058-1073

Schot WD, Brenner E, Smeets JB (2010) Robust movement segmentation by combining multiple sources of information. J Neurosci Methods 187:147-155

Siddiqui A (1995) Object size as a determinant of grasping in infancy. J Genet Psychol 156:345-358

Smeets JB, Brenner E (1999) A new view on grasping. Mot Control 3:237-271

Stelmach GE, Castiello U, Jeannerod M (1994) Orienting the finger opposition space during prehension movements. J Motor Behav $26: 178-186$

Timmann D, Stelmach GE, Bloedel JR (1996) Grasping component alterations and limb transport. Exp Brain Res 108:486-492

Tresilian JR, Stelmach GE (1997) Common organization for unimanual and bimanual reach-to-grasp tasks. Exp Brain Res 115:283-299

van der Kamp J, Savelsbergh GJP (2000) Action and perception in infancy. Infant Behav Dev 23:237-251

van der Kamp J, Savelsbergh GJP, Davis WE (1998) Body-scaled ratio as a control parameter for prehension in 5- to 9-year-old children. Dev Psychobiol 33:351-361

van der Kamp J, Rivas F, van Doorn H, Savelsbergh G (2008) Ventral and dorsal system contributions to visual anticipation in fast ball sports. Int J Sport Psychol 39:100-130

van Doorn H, van der Kamp J, Savelsbergh GJ (2007) Grasping the Muller-Lyer illusion: the contributions of vision for perception in action. Neuropsychologia 45:1939-1947 van Hof P, van der Kamp J, Savelsbergh GJP (2002) The relation of unimanual and bimanual reaching to crossing the midline. Child Dev 73:1353-1362

van Hof P, van der Kamp J, Savelsbergh GJP (2008) The relation between infants' perception of catchableness and the control of catching. Dev Psychol 44:182-194

van Soest AJ, Beek PJ (2010) Similar findings, different interpretations: a response to the commentary by Bootsma (2010). J Exp Psychol Hum Percept Perform 36:1064-1066

van Wermeskerken M, van der Kamp J, Savelsbergh GJP (2010) The early development of the use of visual information for action and perception. In: Elliott D, Khan M (eds) Vision and goal-directed movement: neurobehavioral perspectives. Human Kinetics, Champaign, pp 293-313

van Wermeskerken M, van der Kamp J, te Velde AF, Valero-Garcia AV, Hoozemans MJM, Savelsbergh GJP (2011) Anticipatory reaching of seven- to eleven-month-old infants in occlusion situations. Infant Behav Dev 34:45-54

von Hofsten C, Fazel-Zandy S (1984) Development of visually guided hand orientation in reaching. J Exp Child Psychol 38:208-219

von Hofsten C, Roennqvist L (1988) Preparation for grasping an object: a developmental study. J Exp Psychol Hum Percept Perform 14:610-621

Wallace SA, Weeks DL, Kelso JAS (1990) Temporal constraints in reaching and grasping behavior. Hum Mov Sci 9:69-93

Warren WH, Whang S (1987) Visual guidance of walking through apertures: body scaled information for affordances. J Exp Psychol Hum Percept Perform 13:371-383

Woodworth RS (1899) The accuracy of voluntary movements. Psychol Rev Monogr 3:1-119 